\title{
Editorial
}

\section{Advanced Techniques for Computational and Information Sciences}

\author{
William Guo, ${ }^{1}$ Chih-Cheng Hung, ${ }^{2}$ Paul Scheunders, ${ }^{3}$ Bor-Chen Kuo, \\ Yihua Lan, ${ }^{5}$ and Zhiqiang $\mathrm{Ma}^{6}$ \\ ${ }^{1}$ Central Queensland University, North Rockhampton, QLD 4702, Australia \\ ${ }^{2}$ Kennesaw State University, Marietta, GA 30060, USA \\ ${ }^{3}$ University of Antwerp, 2610 Antwerp, Belgium \\ ${ }^{4}$ National Taichung University of Education, Taichung 403, Taiwan \\ ${ }^{5}$ Nanyang Normal University, Nanyang 473061, China \\ ${ }^{6}$ Northeast Normal University, Changchun 130117, China \\ Correspondence should be addressed to William Guo; w.guo@cqu.edu.au
}

Received 22 November 2015; Accepted 28 December 2015

Copyright (c) 2016 William Guo et al. This is an open access article distributed under the Creative Commons Attribution License, which permits unrestricted use, distribution, and reproduction in any medium, provided the original work is properly cited.

New techniques in computational and information sciences have played an important role in keeping advancing the so called knowledge economy. Advanced techniques have been introduced to or emerging in almost every field of the scientific world for hundreds of years, which has been accelerated since the late 1970s when the advancement in computers and digital technologies brought the world into the Information Era. In addition to the rapid development of computational intelligence and new data fusion techniques in the past thirty years [1-4], mobile and cloud computing, grid computing driven numeric computation models, big data intelligence, and other emerging technologies have not only expanded the scope of traditional simulation and modelling in many scientific and engineering disciplines [5-8] but also enabled the fusion of traditional and contemporary methods in almost every field in the world [9-11].

This special issue is to facilitate dissemination of recent research outcomes resulting from applying innovative and advanced techniques in computational and information sciences to various scientific and engineering disciplines. The papers included in this special issue were selected from submissions to both Mathematical Problems in Engineering (MPE) directly and the 2014 International Conference on Information Technology, Computation and Applications
(ICITCA2014) held in Anyang of China in December 2014, which followed the success of ICITCA2013 [12]. In total, two hundred and sixty-six (266) papers were submitted to this special issue. Contributors were from more than 100 institutions in 25 countries, including Australia, Bulgaria, Canada, China, Czech, Egypt, India, Iran, Japan, Korea, Malaysia, Mexico, Pakistan, Portugal, Saudi Arabia, South Africa, Taiwan, Thailand, Tunisia, Turkey, UAE, Ukraine, UK, USA, and Uzbekistan. After rigorous peer review of each of these submissions, thirty-nine revised papers were accepted for publishing in this special issue. Depending on the completion time of revision and final editing, these papers have been published progressively since September 2015.

Categorically, there are 15 papers in the broad area of digital audio, video, and image processing and pattern recognition. S. Zhao et al. presented a variational Bayesian superresolution approach using adaptive image prior model. C.-H. Hsieh and J.-D. Lee compared the markerless augmented reality and stereo video see-through head-mounted display device. L.-Y. He et al. proposed a hardy variation framework for restoration of weather degraded images. W. Wang et al. studied dehazing multiscale single image based on adaptive wavelet fusion. M. Liang et al. investigated a learning-based video superresolution reconstruction using 
spatiotemporal nonlocal similarity. M. A. Nematollahi et al. proposed a robust digital speech watermarking for online speaker recognition, whereas X. Zhang et al. used an infinite student's $t$-mixture model for robust speech emotion recognition. Z. Jian et al. presented a new method of image watermarking based on extended discrete Shearlet and insertion in horizontal cone using the largest information entropy. C. Ruan et al. combined WT with ICA to create a new image denoising method. Y. Feng et al. described a method of accurate modulation recognition for QPSK signals. P. $\mathrm{Li}$ and W. Ge proposed a soft shadow detection method based on MRF for remote sensing images. T. Yuan et al. studied subtropical forestry index retrieval using terrestrial Laser scanning and hemispherical photography. A color texture image segmentation method based on fuzzy c-means clustering and region-level Markov random field model was presented by G. Liu et al., whereas a new means of automatic recognition of Chinese person name was described by C. Gu et al. A method of extracting vision-based faint vibration using singular value decomposition was discussed by X. Lei et al.

The second largest group of 11 papers is around data mining techniques and applications. Areas covered include community detection in complex networks (by B. Xu et al.), bias modelling relation extraction (by Y. Xiang et al.), error checking for Chinese query (by J. Duan et al.), traffic flow forecasting (by Z. Yu et al.), diffusion of vector field for active contour model (by G. Liu et al.), semantic similarity measure considering multi-inheritance in biomedicine (by F. Yang et al.), meteorological data analysis (by Z. Meng et al.), Chinese character and sentence editing and analysis (by Q. Li and X. Li and Y. Liu and H. Sun), and evaluation on system performance (by N. Yang et al.).

Optimization and applications were reported in 7 articles. Techniques used in these studies include particle swarm optimization (S. Goudarzi et al. and M. Jakubcová et al.), fuzzy neural networks (W. Lei et al.), genetic algorithms (Y. Deng et al.), immersed boundary method (Mizuno et al.), Bayesian prediction model (Z.-L. Xiang et al.), and constrained optimization by position and tangent vector (G. Hu et al.).

There are four papers in adaptive control and automation for various systems, such as block preconditioners for complex symmetric linear systems (S.-L. Wu and C.-X. Li), EIV-based interference alignment scheme (Z. Kong et al.), machine vision-based automatic detection (J. Chi et al.), and decentralized control for large-scale interconnected nonlinear systems (T. Guo). Two papers were around information system design and management in mobile or cloud environment (Y.-Q. Zhao and J. Hu et al.).

All these papers have made new contributions to the broad areas of computational and information sciences.

\section{Acknowledgments}

We are grateful to all authors and reviewers for their contributions to this special issue. The organizers of ICITCA2014 are thanked for their consistent support during the entire journey.

\author{
William Guo \\ Chih-Cheng Hung \\ Paul Scheunders \\ Bor-Chen Kuo \\ Yihua Lan \\ Zhiqiang $\mathrm{Ma}$
}

\section{References}

[1] J. M. Zurada, R. J. Marks, and C. J. Robinson, Eds., Review of Computational Intelligence: Imitating Life, IEEE Press, Piscataway, NJ, USA, 1994.

[2] L. Rutkowski, Computational Intelligence: Methods and Techniques, Springer, New York, NY, USA, 2008.

[3] A. Siddique and H. Nazmul, Computational Intelligence: Synergies of Fuzzy Logic, Neural Networks and Evolutionary Computing, John Wiley \& Sons, Hoboken, NJ, USA, 2013.

[4] L. Liao and W. W. Guo, "Incorporating utility and cloud theories for owner evaluation in tendering," Expert Systems with Applications, vol. 39, no. 5, pp. 5894-5899, 2012.

[5] D. Moreno-Salinas, D. Chaos, E. Besada-Portas, J. A. LópezOrozco, J. M. de la Cruz, and J. Aranda, "Semiphysical modelling of the nonlinear dynamics of a surface craft with LSSVM," Mathematical Problems in Engineering, vol. 2013, Article ID 890120, 13 pages, 2013.

[6] N. Li, W. Yi, M. Sun, and G. Gong, "Development and application of intelligent system modeling and simulation platform," Simulation Modelling Practice and Theory, vol. 29, pp. 149-162, 2012.

[7] C.-C. Hung, S. Kulkarni, and B.-C. Kuo, "A new weighted fuzzy C-means clustering algorithm for remotely sensed image classification," IEEE Journal on Selected Topics in Signal Processing, vol. 5, no. 3, pp. 543-553, 2011.

[8] R. Heylen and P. Scheunders, "Multidimensional pixel purity index for convex hull estimation and endmember extraction," IEEE Transactions on Geoscience and Remote Sensing, vol. 51, no. 7, pp. 4059-4069, 2013.

[9] W. W. Guo, "A novel application of neural networks for instant iron-ore grade estimation," Expert Systems with Applications, vol. 37, no. 12, pp. 8729-8735, 2010.

[10] M. Mahi, Ö. K. Baykan, and H. Kodaz, "A new hybrid method based on Particle Swarm Optimization, Ant Colony Optimization and 3-Opt algorithms for Traveling Salesman Problem," Applied Soft Computing, vol. 30, pp. 484-490, 2015.

[11] M. Chen and J. Yu, "Disturbance observer-based adaptive sliding mode control for near-space vehicles," Nonlinear Dynamics, vol. 82, no. 4, pp. 1671-1682, 2015.

[12] W. Guo, C.-C. Hung, R. Shepherd, Z. Ma, and S. Kulkarni, "Intelligent techniques for simulation and modelling," Mathematical Problems in Engineering, vol. 2014, Article ID 179457, 3 pages, 2014. 


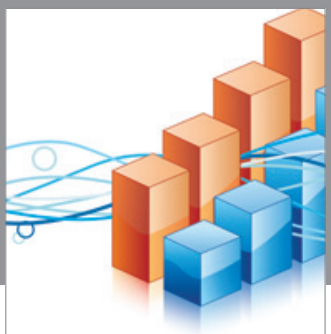

Advances in

Operations Research

vatem alat4



\section{The Scientific} World Journal

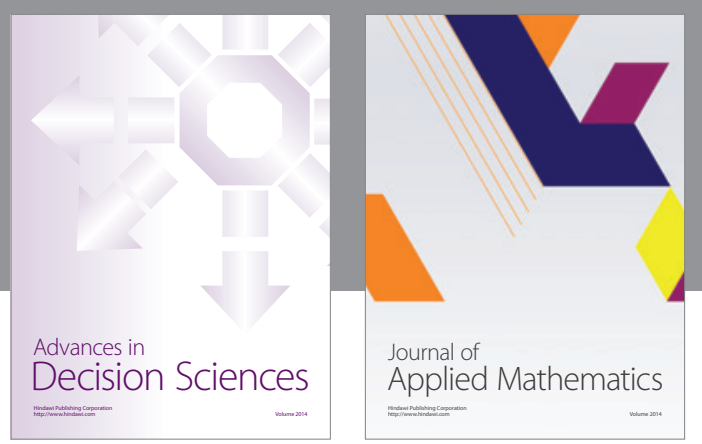

Algebra

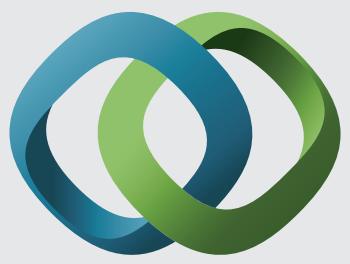

\section{Hindawi}

Submit your manuscripts at

http://www.hindawi.com




Mathematical Problems in Engineering
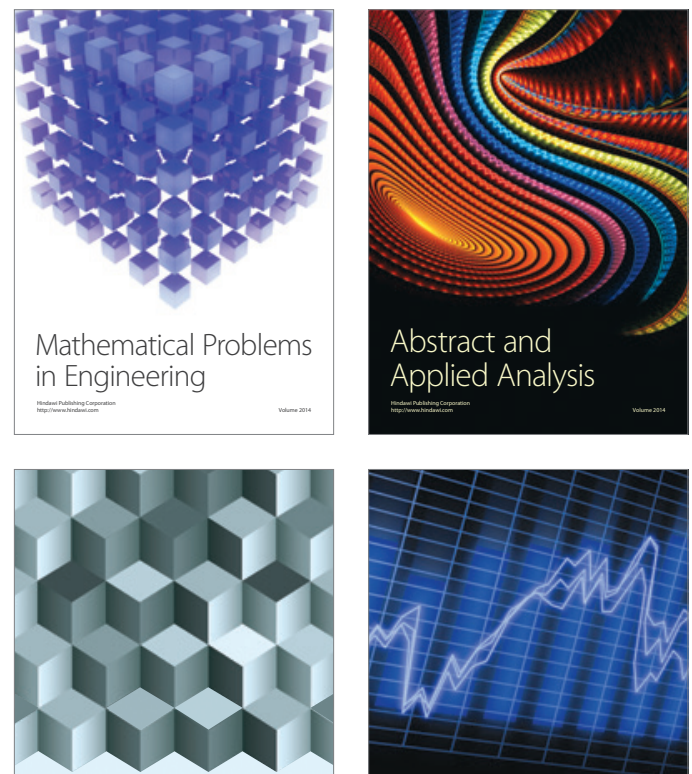

Journal of

Function Spaces

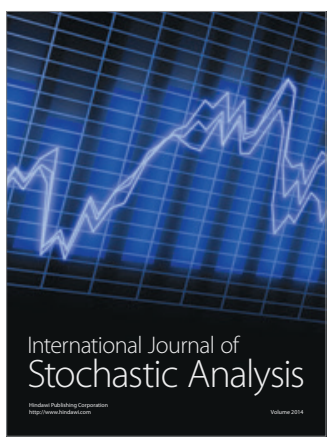


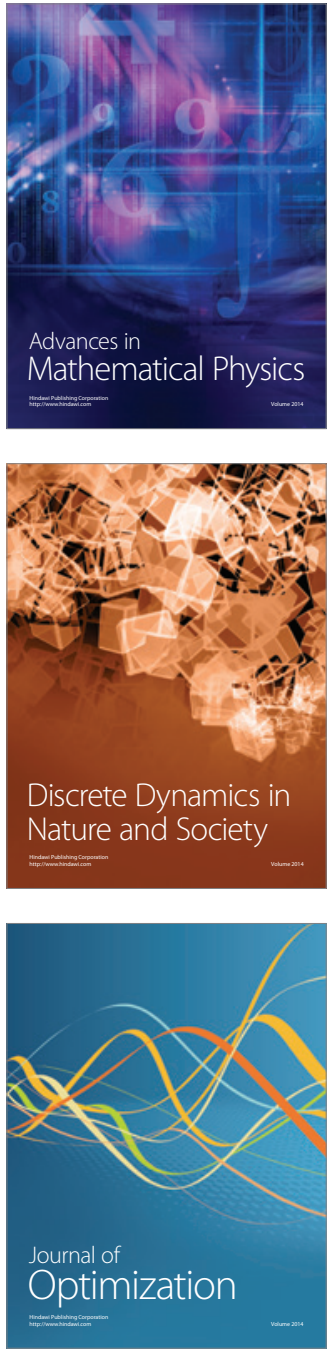